\title{
A case series of craniotomy in obstetric patients: a lifesaving treatment modality
}

\section{S. S. Subha*, Mohana Dhanapal, Aiswary}

Department of Obstetrics and Gynecology, Government Mohan Kumaramangalam Medical College, Salem, Tamil Nadu, India

Received: 19 February 2018

Accepted: 27 March 2018

\section{*Correspondence:}

Dr. S. S. Subha,

E-mail: drppsamysubha@gmail.com

Copyright: (c) the author(s), publisher and licensee Medip Academy. This is an open-access article distributed under the terms of the Creative Commons Attribution Non-Commercial License, which permits unrestricted non-commercial use, distribution, and reproduction in any medium, provided the original work is properly cited.

\begin{abstract}
Neurosurgical disorders are a significant cause of non-obstetric death and disability in pregnant women. They pose a unique therapeutic challenge. The changes in normal physiology during pregnancy add to the complexity of management. The common conditions encountered are intracranial hemorrhage, tumours, trauma and spinal pathologies. It is preferable to wait until the third trimester if the indication is semi-elective. Here we present a prospective study of four cases, managed with neurosurgery in pregnancy and puerperium. Of the 4 cases two cases were craniotomy done for cortical vein thrombosis and two cases of excision of space occupying lesions. Pregnancy itself should not be considered a contra-indication to neurosurgery, which when considered early in some patients, proves to be life-saving.
\end{abstract}

Keywords: Maternal morbidity, Neurosurgery, Pregnancy

\section{INTRODUCTION}

As the maternal mortality from obstetric causes is decreasing nowadays, due to increased understanding of the etiologies and management, the non-obstetric causes of maternal mortality are on the rise, and neurological diseases contribute a major share. ${ }^{1}$ Most neurological manifestations in pregnancy like cerebral venous thrombosis, mild haemorrhages and minimal trauma, small tumours are managed conservatively or with medical treatments, mostly anticoagulants and steroids. ${ }^{2-4}$ But due to the physiological changes in pregnancy and hormonal effects on some neurological disorders, medical management may not be sufficient, resort to neurosurgery in such cases may be lifesaving. ${ }^{5}$ The major worries we face are the maternal outcomes after neurosurgery, the pregnancy changes affecting outcomes, fetal complications that may occur due to the surgery or the anaesthesia during surgery, and hence the dilemma in decision making whether to proceed with the surgical treatment or not. Despite all these considerations, neurosurgery may be lifesaving in many instances, and hence pregnancy itself must not be considered a contraindication for surgery. The advance in anesthesia and surgical techniques have improved the outcomes of the surgical management.

\section{CASE REPORT}

\section{Case 1}

Mrs. X, 34 years old, G2A1, married since 1 year, with regular menstrual cycles, term gestation, booked and immunized at a private hospital, already diagnosed as fibroid complicating pregnancy, was admitted with complaints of abdominal pain with oblique lie of the 
fetus. Emergency caesarean section done, to deliver an alive boy baby. The patient complained of weakness of right upper limb and lower limb followed by seizures and headache on postoperative day 10. Computerized Tomography-brain done showed hyperdense lesion noted in left frontal lobe with surrounding edema. Magnetic Resonance Imaging brain done-showed dural venous sinus thrombosis (Figure 1). Patient was started on Injections Mannitol $175 \mathrm{ml}$ once daily, Phenytoin $100 \mathrm{mg}$ intravenous thrice daily and heparin-unfractionated 5000 units subcutaneous twice daily.

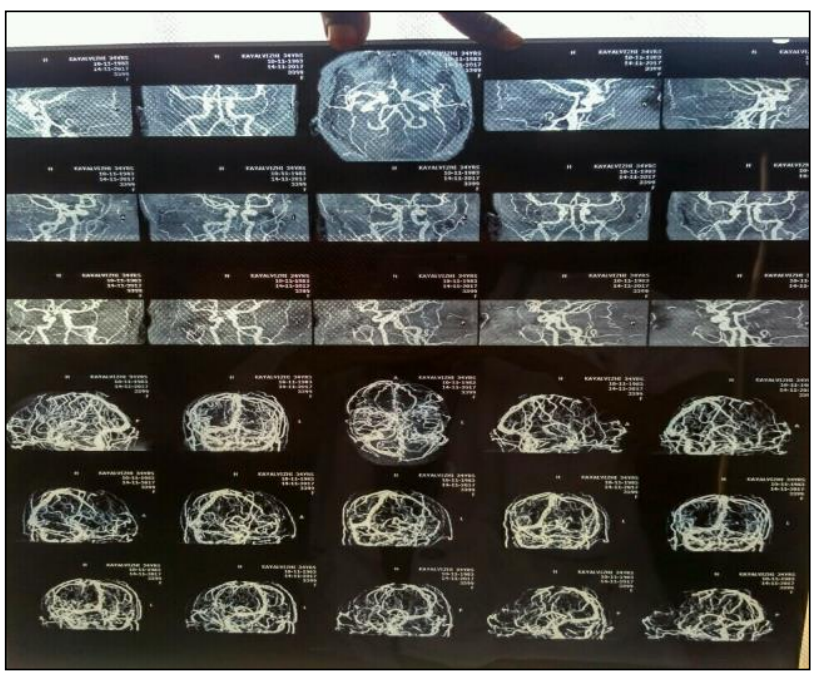

Figure 1: MRV dural venous sinus thrombosis.

On the next day, patient developed altered sensorium and became drowsy. CT-Brain repeated showed left parietal lobe edema with mass effect, hemorrhagic infarct and no midline shift. Heparin was stopped, and neurosurgeon advised conservative management as there was no midline shift.

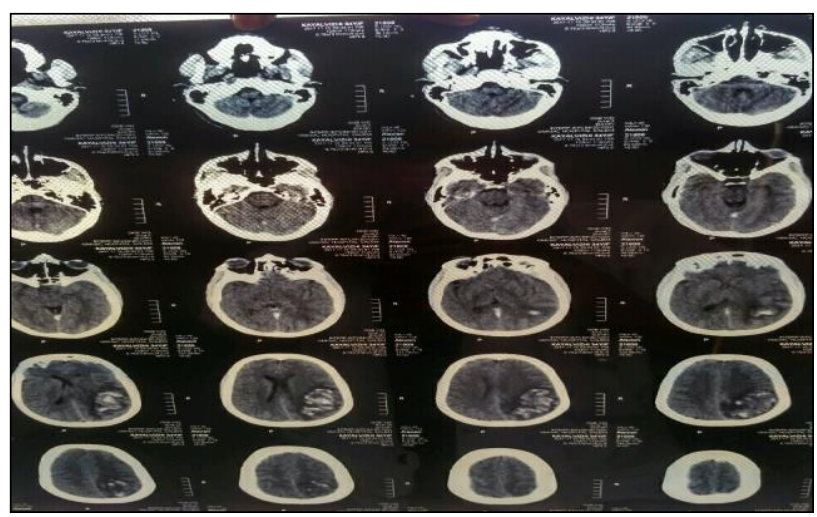

Figure 2: Hemorrhagic infarct of left parietal lobe with mass effect and midline shift.

Cardiac evaluation done, and ECHO done was normal. Fundus examination showed early papilledema. Two days later, POD-13, patient became unconscious and was intubated and initiated on mechanical ventilation. CTBrain repeated showed left parietal mixed dense lesion with mass effect and midline shift (Figure 2), and the patient was planned for emergency decompression surgery, in view of CVT with hemorrhagic transformation.

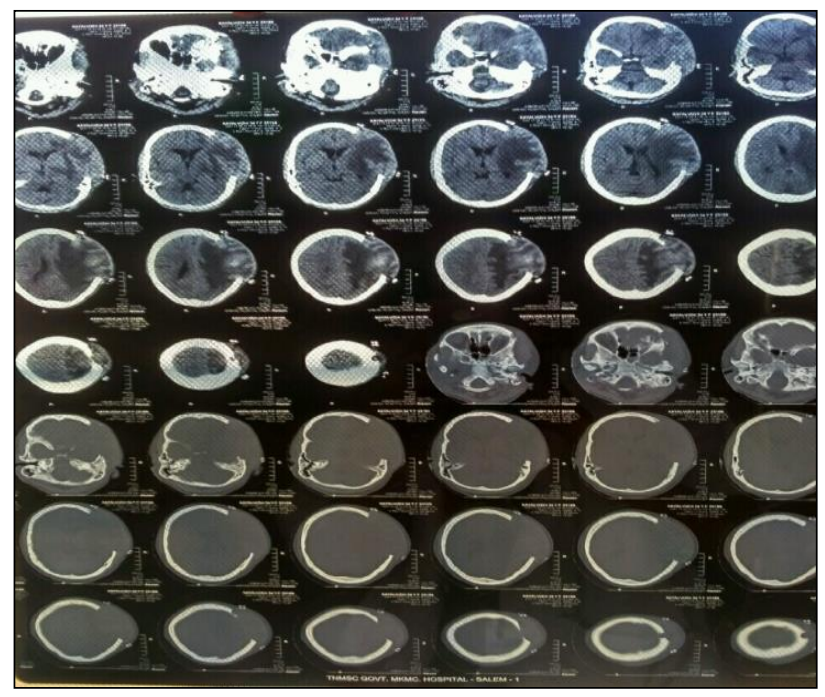

Figure 3: Postoperative CT after decompressive FTP craniectomy.

Left fronto-tempero-parietal decompressive craniectomy done. Trauma flap incision made and myocutaneous flap raised. Craniotomy done with six burr holes. Bone flap raised and discarded. Dura opened sylvian based. Intraoperatively, the brain was found to be tense with parietal contusion. Hemostasis secured. Dura replaced. Galea and skin closed. Patient shifted to Intensive Care Unit postoperatively and monitored. She was restarted on heparin and other drugs postoperatively. Post-operatively, CT-Brain showed postoperative changes, with no other significant changes or midline shift (Figure 3). Tracheostomy was done, physiotherapy given.

Patient recovered well and regained consciousness and muscle power. She was gradually weaned from supports and maintained her vitals. Patient was changed over to warfarin from heparin and to oral phenytoin and antihypertensives. She was discharged and advised regular follow-up at OG and neurology OPD.

\section{Case 2}

This is a similar case like the previous one, where decompressive craniotomy proved lifesaving in postpartum cerebral venous thrombosis. Mrs. X, 24 years old, P1L1, delivered an alive boy baby in a PHC, and on postnatal day 15 had complaints of giddiness, headache and vomiting. Patient was referred to a nearby GH and there became unconscious. She was referred to our institution in a state of unconsciousness as cortical vein thrombosis, for tertiary care.

On admission patient was drowsy, responding to painful stimuli only, with right hemiparesis. Her vitals were 
stable and systemic examination of cardiovascular and respiratory systems were normal.

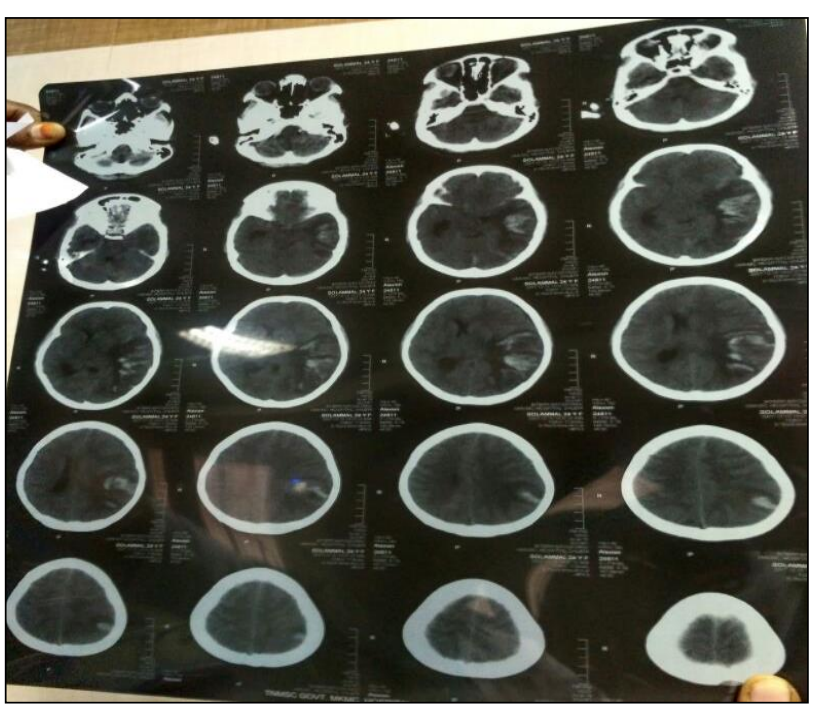

Figure 4: CT brain showing left sided temperoparietal hemorrhagic infact with mass effect and midline shift.

Her CT-Brain showed left sided temporal and parietal hemorrhagic infarct with mass effect and midline shift (Figure 4). Medical management with frusemide, mannitol and heparin started immediately.

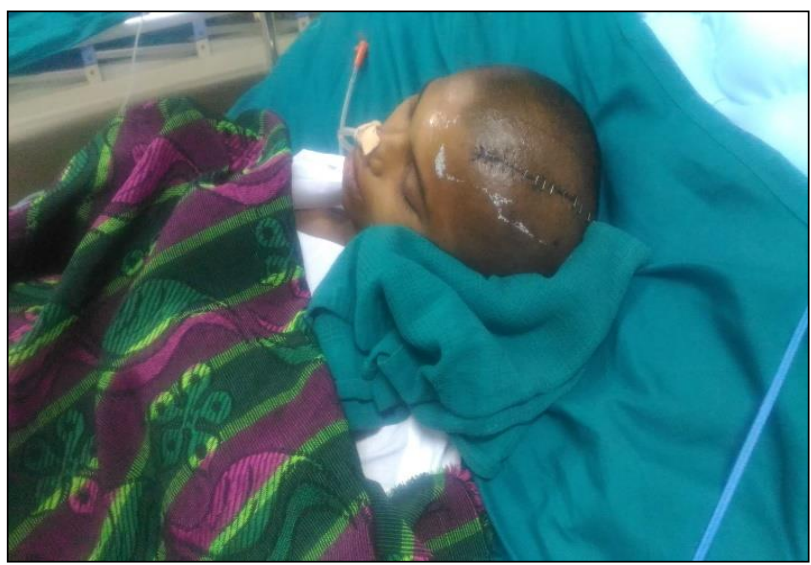

Figure 5: Postoperative picture after left frontotempero-parietal decompressive craniotomy.

She was then planned for emergency decompressive craniotomy. Proceeded with left fronto-tempero-parietal decompressive craniotomy. Under general anesthesia, patient in supine position, FTP trauma flap raised. Eight burr holes FTP craniotomy done, and free bone flap removed. Temporal bone decompression done. Antiedema measures given. Postoperatively patient shifted to intensive care unit and monitored (Figure 5). Ventilator support maintained for three days postoperatively and weaned. Anti-edema drugs, antiepileptics, anticoagulants and higher antibiotics given. Patient recovered well and was discharged.

\section{Case 3}

25 Years old Mrs. X, G2P2L1, previous 2 cesarean section, 20 weeks geatational age, booked and immunized at a PHC, belonging to socioeconomic status class of $\mathrm{V}$, referred as a case of neurofibromatosis with headache and seizures for further evaluation. She had history of seizures from 3 years to 10 years of age, had 3 episodes, not on treatment, not evaluated further. Her mother also has multiple neurofibromas all over the body (Figure 6 and 7. On admission, patient was conscious, oriented, vitals stable, systemic examination was normal, and local examination revealed multiple neurofibromas seen all over the body and Café au lait spots (Figure 6).

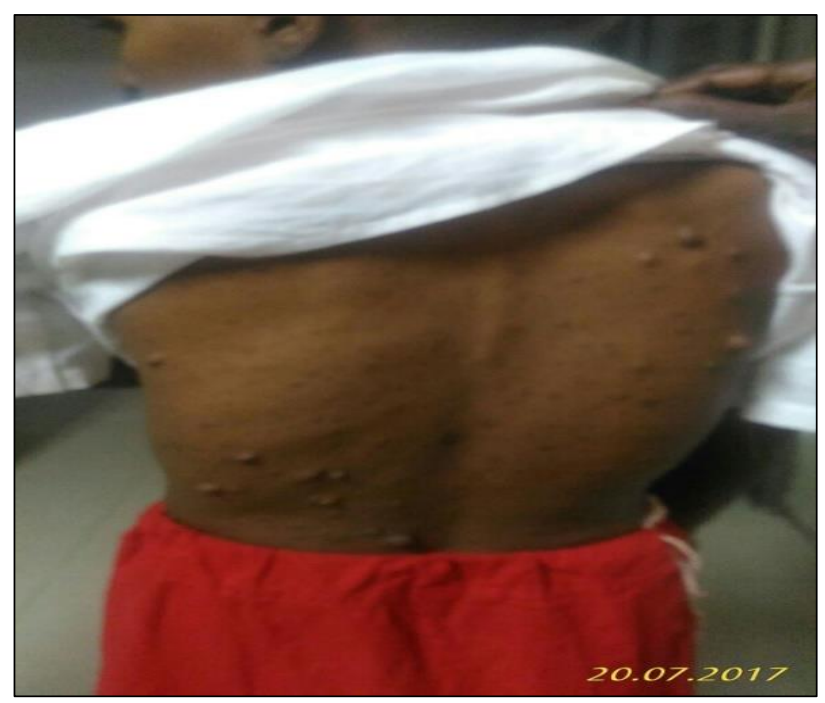

Figure 6: Multiple neurofibromas and Café au lait spots.

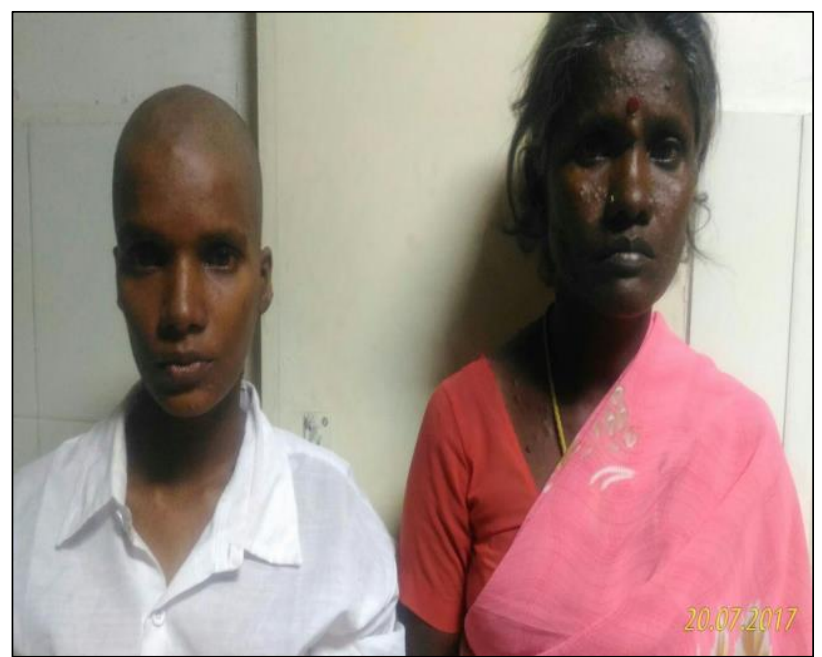

Figure 7: Neurofibromatosis in both the patient and her mother.

MRI brain showed well defined cystic lesion with mural nodule in Right frontal lobe measuring about $76 \mathrm{~mm} \times 44$ $\mathrm{mm} \times 40 \mathrm{~mm}$, crossing midline, herniates to Lt frontal 
lobe, surrounding vasogenic edema, midline shift $16 \mathrm{~mm}$, suggestive of acute obstructive hydrocephalus (Figure 8).

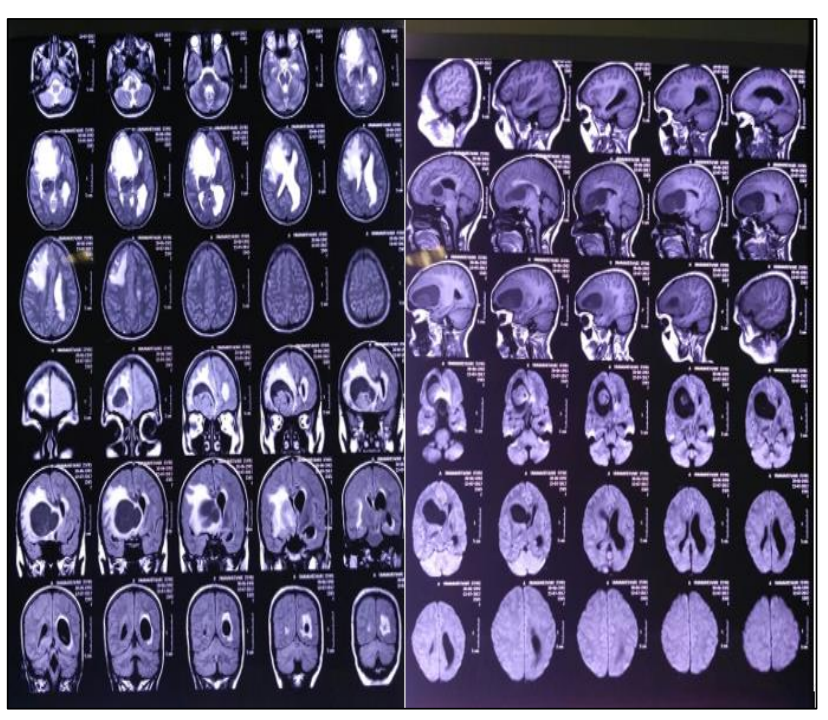

Figure 8: MRI brain showing space occupying lesion with obstructive hydrocephalus.

Right frontal craniotomy and total excision of the space occupying lesion was done under general anesthesia. Right hemi cranial skin incision made, sub galeal skin flaps raised and pericranial flaps raised, 4 burholes made, right frontal craniotomy made. Dura opened, superior sagittal sinus based corticetomy done, cystic fluid aspirated about $20 \mathrm{ml}$.

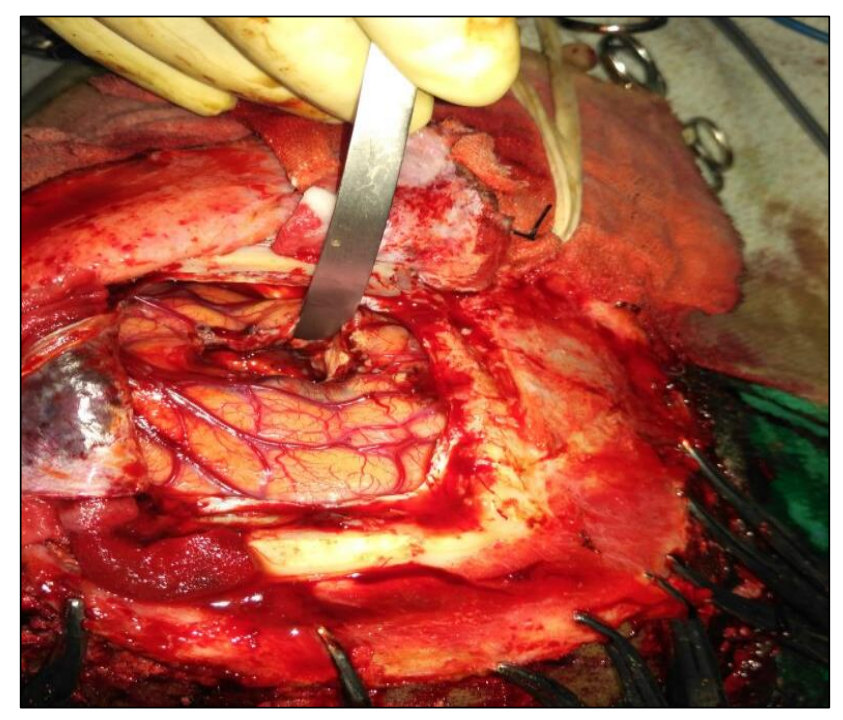

Figure 9: Intra operative picture of space occupying lesion after raising bone flap.

Large greyish blue mural nodule was found, (Figure 9) total excision of mural nodule was done, cyst wall was completely removed. Complete haemostasis obtained, surgical layering done, flaps replaced. Continuous fetal heart rate monitoring was done throughout the procedure (Figure 10).

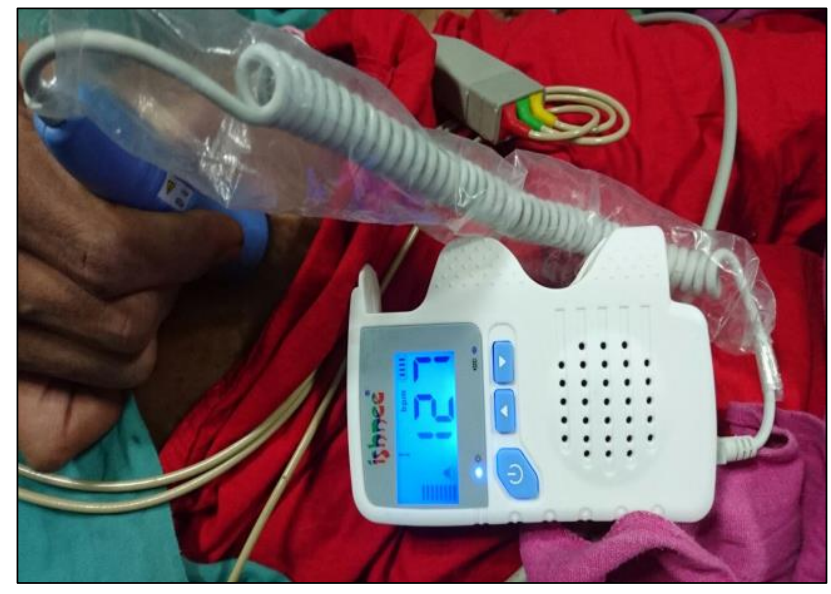

Figure 10: Continuous fetal heart rate monitoring was done throughout the procedure.

\section{Case 4}

Mrs. X, 20 years old, primi, 30 weeks gestational age, booked and immunized at a PHC, referred from the PHC as a case of focal seizures. She was normal since childhood and recently developed focal seizures involving the left upper limb and lower limb for two weeks duration on and off, for which she was on irregular treatment in a private hospital. On referral to our hospital patient was conscious, oriented, vitals stable, systemic examination of the cardiovascular and respiratory systems were normal. She had recurrent episodes of focal seizures involving the left upper and lower limb. MRI Brain showed right parietal mass lesion with massive edema. Neurologist opinion obtained, and patient was started on antiedema drugs and anti-epileptics.

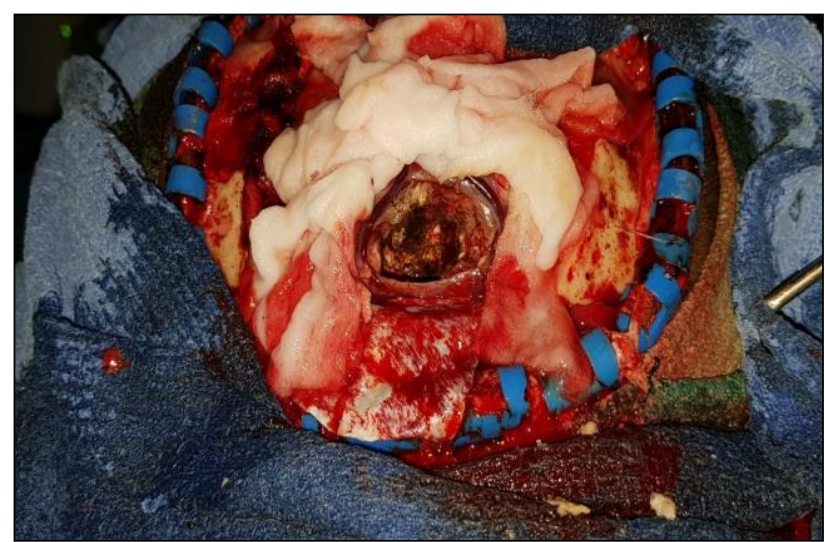

Figure 11: Intra operative picture of the glioma.

Neurosurgeon opinion obtained as MRI Brain features suggestive of glioma and planned for craniotomy and excision of the space occupying lesion. Patient assessed and electively posted for right parietal craniotomy and excision of the SOL. Under general anesthesia, patient in supine position with a slight tilt to left side, right side parietal horse shoe flap made. Four burr hole craniotomy done, and free flap raised. In the posterior parietal region 
at a depth of $1 \mathrm{~cm}$, tumour identified-firm, greyish white and vascularized mass in gross appearance (Figure 11). Total excision of the mass done. Intraoperatively, meticulous fetal monitoring done throughout the procedure. Postoperatively, patient monitored in the intensive care unit, and put on anti-epileptics, antibiotics, steroids. Vitals were monitored, and postoperative period was uneventful. Close Fetal monitoring done.

\section{DISCUSSION}

Pathology of the central nervous system remains an important indirect cause of maternal mortality with incidence around 30-40 deaths per triennium. Intracranial hemorrhage and subarachnoid hemorrhages account for majority of cases causing deaths, where neurosurgical intervention can be provided. ${ }^{6}$ The other causes needing neurosurgery in pregnancy are due to trauma, primary central nervous system tumors, spinal pathologies and ventriculo-peritoneal shunts. SAH occurs in 10-20 per $1,00,000$ pregnancies and has devastating consequences. They present as severe thunderclap headache and have varied sequelae like cranial nerve lesions to unconsciousness.

Most common cause of SAH is rupture of intracranial aneurysm, and the incidence of aneurismal rupture is increased in pregnancy., ${ }^{7,8}$ Trauma complicates about 6$7 \%$ pregnancies and is an important cause of indirect maternal death and may well have cranial and spinal injuries requiring neurosurgery. ${ }^{9,10}$ The incidence of central nervous system tumours in pregnancy is about 6 per $100000 .^{11}$

Gliomas are the most common malignant tumours presenting during pregnancy. Some tumours like meningiomas, which are the most common benign tumours in pregnancy, express estrogen or progesterone receptors and may increase in size during pregnancy. Certain pituitary tumours with excess adrenocorticotropic hormone or thyrotropic hormone production may require excision during pregnancy. Spinal problems requiring neurosurgery in the antenatal period may be symptomatic disc herniation, which is more common in pregnancy, due to the effects of relaxin. Other causes may be vertebral canal hematoma and vertebral abcess.

Pregnant women presenting for non-obstetric surgery represent a unique clinical situation, where the health of the mother is paramount, but equally careful consideration needs to be given to the fetus. They are mostly performed in the second trimester. After the period of viability, three options for surgery include 1 . Caesarean section proceeded by neurosurgery as a continuous process 2. Caesarean section with neurosurgery at a later date 3 . maintaining pregnancy and proceeding with neurosurgery. The management of symptomatic brain tumours depend upon the hemodynamic stability of the patient and the gestational age. In a hemodynamically unstable patient, we always proceed with the neurosurgery and then deliver by caesarean section, when the fetus has crossed viability. In a stable patient, we can observationally wait until the period of viability and then deliver the patient vaginally or by caesarean section, then later proceed with the neurosurgery.

Here we present a series of four cases who underwent neurosurgery- two in the antenatal period for space occupying lesions and two cases for postpartum cerebral venous thrombosis. All four patients, operated at the correct time, and monitored well postoperatively had very good clinical outcomes.

\section{CONCLUSION}

The requirement for neurosurgery in the parturient is a rare occurrence. When these patients do present, however, their care is likely to be complex and challenging. As such a multidisciplinary approach should be adopted throughout their period of care. Protocols should be developed for emergency neurosurgical treatments and with established lines of communication between specialities, these surgeries may prove to be lifesaving.

\section{Funding: No funding sources \\ Conflict of interest: None declared \\ Ethical approval: Not required}

\section{REFERENCES}

1. Kapoor, Dipanwita, Wallace S. Trends in maternal deaths from epilepsy in the United Kingdom: a 30year retrospective review. Obstet Med. 2014;7.4:1604.

2. Agostoni E, Aliprandi A, Longoni M. Cerebral venous thrombosis. Expert Rev Neurother. 2009;9(4):553-64.

3. Bates S, Greer I, Hirsh J, Ginsberg J. Use of antithrombotic agents during pregnancy: the seventh ACCP conference on antithrombotic and thrombolytic therapy. Chest. 2004;126(3):627S-44S.

4. Medel R, Monteith SJ, Crowley RW, Dumont AS. A review of therapeutic strategies for the management of cerebral venous sinus thrombosis. Neurosurg Focus. 2009;27(5):E6.

5. Coutinho JM, Majoie CB, Coert BA, Stam J. Decompressive hemicraniectomy in cerebral sinus thrombosis: consecutive case series and review of the literature. Stroke. 2009;40(6):2233-5.

6. Centre for Maternal and Child Enquiries (CMACE). Saving mothers' lives: reviewing maternal deaths to make motherhood safer: 2006-08. The eighth report on confidential enquiries into maternal deaths in the United Kingdom. BJOG. 2011;118(1):1-203.

7. Dias MS, Sekhar LN. Intracranial hemorrhage from aneurysms and arteriovenous malformations during pregnancy and the puerperium. Neurosurg. 1990;27:855-65. 
8. Hunt HB, Schifrin BS, Suzuki K. Ruptured berry aneurysms and pregnancy. Obstet Gynecol. 1974;:43:827-37.

9. Shah AJ, Kilcline BA. Trauma in pregnancy. Emerg Med Clin N Am. 2003;21:615-29.

10. Fast A, Shapiro D, Ducommun EJ, Friedmann LW, Bouklas T, Floman Y. Low back pain in pregnancy. Spine. 1987;12:368-71.
11. Wang LP, Peach MJ. Neuroanesthesia for the Pregnant Woman. Neurosurg Anesthesiol. 2008;107(1):193-200.

Cite this article as: Subha SS, Dhanapal M, Aiswary. A case series of craniotomy in obstetric patients: a lifesaving treatment modality. Int J Reprod Contracept Obstet Gynecol 2018;7:2055-60. 